\title{
Labyrinthe
}

$24 \mid 2006$ (2)

Faut-il être postcolonial?

\section{Traversées de frontières : postcolonialité et études de « genre » en Amérique}

Entretien réalisé par Grégoire Leménager et Laurence Marie

\section{Anne Berger}

\section{OpenEdition}

\section{Journals}

Édition électronique

URL : http://journals.openedition.org/labyrinthe/1245

DOI : $10.4000 /$ labyrinthe. 1245

ISSN : 1950-6031

Éditeur

Hermann

Édition imprimée

Date de publication : 15 juin 2006

Pagination : 11-37

ISBN : 2-9526131-1-7

Référence électronique

Anne Berger, «Traversées de frontières : postcolonialité et études de "genre » en Amérique »,

Labyrinthe [En ligne], 24 | 2006 (2), mis en ligne le 24 juillet 2008, consulté le 30 avril 2019. URL : http:// journals.openedition.org/labyrinthe/1245; DOI : 10.4000/labyrinthe.1245

Propriété intellectuelle 


\title{
TRAVERSÉES DE FRONTIÈRES : postcolonialité et études de «genre» en Amérique
}

\author{
Entretien avec Anne Berger
}

Propos recueillis par Grégoire LEMÉNAGER et Laurence MARIE

Et si, plutôt que de prétendre saisir le sens fuyant de ce terme en vogue, postcolonial, il fallait l'approcher à tâtons, en examinant le terreau qui a favorisé son éclosion? Autrement dit, commencer par considérer les postcolonial studies comme des studies : parmi d'autres, parents éloignés ou proches cousines, qui dessinent en se croisant et s'opposant un panorama touffu dont le public français n'est guère familier. Vues d'ici, en effet, qu'ont à voir les catégories gender, queer, subaltern, transnational et autres Afro-American avec la théorie postcoloniale?... On peut à bon droit ironiser sur le génie lexical américain, qui pousse les universitaires à l'invention permanente de nouveaux labels, dans la surenchère d'un politiquement correct qui est, aussi, économique à bien des égards. Mais l'ironie n'interdit pas la curiosité. On peut vouloir comprendre à quoi, et surtout avec quoi riment ces produits intellectuels qui, aujourd'hui, s'exportent et se disséminent mondialement - contribuant ainsi d'une façon ou d'une autre à forger ce que seront les grandes pensées du XXI siècle. Or, sans négliger cette expansion vaste et diffuse, il reste difficile de ne pas rapporter les dimensions épistémologiques et politiques des studies au champ universitaire spécifique où, en relation avec l'évolution de la société américaine, elles se sont développées et continuent à le faire. Car les frontières que la théorie postcoloniale invite à déplacer sont peut-être, d'abord, présentes à ce niveau-là. Et leur subversion suppose inévitablement le tracé de nouvelles lignes de démarcation dans les champs du savoir. Quelques-unes des frontières de la théorie postcoloniale : voici en somme ce qu'il s'est agi là d'esquisser.

Pour évoquer ces questions, que nous souhaitions aussi sommaires que nécessaires, Anne Berger est apparue comme une interlocutrice privilé- 
giée. Peu de personnes, en effet, qui soient plus familières de la traversée des frontières géographiques et intellectuelles. Professeur de littérature française à l'université de Cornell, dans l'état de New York, où elle a codirigé un temps, avec l'historien Steve Kaplan, le programme interdisciplinaire d'études françaises et francophones, Anne Berger reste également professeur associée au Centre de recherches en études féminines de Paris VIII (où elle a soutenu sa thèse de doctorat en 1990 et sa thèse d'habilitation en 1999). Son enseignement comme ses travaux de recherche concernent les domaines les plus variés, depuis la littérature des Lumières jusqu'à la poésie moderne, en passant par les politiques culturelles du Maghreb, et les critiques psychanalytiques et féministes. Quelques-uns de ses cours les plus récents portent ainsi sur les rapports entre poésie et pauvreté, les écritures féminines au XIX ${ }^{\mathrm{e}}$ siècle, ou encore les relations entre exotisme et érotisme à travers l'étude des figures de l'Autre dans la littérature française du XVIII' siècle. Auteur de plus d'une cinquantaine d'articles portant sur ces divers sujets (et publiés dans Romantisme, Littérature, Po\&sie, Études françaises, New Literary History, Diacritics, Les Cahiers de l'Herne...), elle a également dirigé la publication de Algeria in Other's Languages (Cornell University Press, 2002) et écrit plusieurs ouvrages, dont Le Banquet de Rimbaud (Champ Vallon, 1992) et Scènes d'aumône. Misère et Poésie au XIXe siècle (Champion, 2004). Elle rédige actuellement un livre consacré à la présence des gender studies sur la scène franco-américaine. Elle est par ailleurs membre du conseil de rédaction de Traces, revue multilingue de théorie culturelle.

LABYRINTHE - Qu' elles s'occupent de questions communautaires ou identitaires, toutes les studies semblent au fond reposer sur un même principe : celui de l'étude d' une «culture » tenue pour minoritaire, c' està-dire délaissée par les humanités traditionnelles. Selon une première approche, peut-on considérer qu' elles se distinguent avant tout par leurs objets d'études respectifs?

ANNE BERGER - Ce que vous dites est un bon point de départ, mais n'est pertinent que jusqu'à un certain point. Chronologiquement, il faut en fait repartir de la guerre froide, à partir de laquelle se sont développés des programmes de recherches constitués autour d'aires géocultu- 
relles : les area studies. Ces programmes sont nés sous l'impulsion du gouvernement fédéral, soucieux de favoriser la formation d'experts compétents en langues et cultures étrangères, et capables de le renseigner sur telle ou telle région du monde. C'est pourquoi les area studies ont été, en leur début au moins, en partie subventionnées par le gouvernement américain. Les études menées dans ce cadre étaient et demeurent très sérieuses, mais leur double visée - « stratégique » ou politique autant qu' « épistémologique »- en fait des domaines problématiques : c'est une parfaite illustration de la collusion décrite par Michel Foucault entre savoir et pouvoir. Une discipline ne se contente pas d'étudier son objet : elle l'invente en même temps - et agit sur lui. C'est particulièrement vrai des area studies, qui ont contribué à produire les découpages régionaux et les aires culturelles ou géopolitiques qu'elles se donnaient pour tâche d'étudier, selon des critères qui relèvent autant de l'idéologie que de la science. Ainsi, par exemple, des South-East Asian studies, ou des Middle-Eastern studies. Les area studies ont servi en ce sens à établir une carte du monde vu de l'Occident. Cela n'a pas empêché des anthropologues, des historiens, des political scientists, d'accomplir un vrai travail dans ce cadre. Mais ils ont progressivement été amenés à s'interroger sur les principes qui président à la constitution de ces aires de recherche et sur le sens de leurs frontières. La critique des études «orientalistes » formulée par Edward Said, à la fin des années 1970, s'inscrit dans ce contexte. Certes, l'essentiel de son livre Orientalism, publié pour la première fois en 1978 et réédité de nombreuses fois ${ }^{1}$, concerne les discours orientalistes européens des XVIII et ${ }^{\mathrm{X} I X}{ }^{\mathrm{e}}$ siècles. Said a voulu montrer que la constitution progressive d'un domaine d'études orientalistes accompagnait et servait à justifier l'expansion coloniale européenne. Mais à la fin de son ouvrage, il indique clairement que l'Amérique impérialiste a pris le relais de l'Europe colonialiste dans ce domaine. Son analyse, d'inspiration foucaldienne, est une analyse des discours, plutôt que des institutions qui les relaient et les étayent. Mais ce qu'il dit revient à mettre en question les principes fondateurs des area studies. C'est dans cette perspective qu'il dénonce longuement les travaux de Bernard Lewis, le grand «expert» américain du MoyenOrient et du monde musulman.

1. New York, Vintage Books, 1978. 
LABYRINTHE - Les études postcoloniales - dont Said est considéré comme l'initiateur - seraient donc nées de la contestation de ces area studies...

ANNE BERGER - En partie, oui. La postcolonial theory conteste l'idée qu'il y a des « aires culturelles » bien délimitées, stables et homogènes ; mais en même temps, c'est plus complexe. D'abord, les area studies n'ont pas été supprimées, ni remplacées ; elles demeurent des réalités institutionnelles et des pôles d'attraction forts dans le paysage universitaire américain contemporain. En particulier, elle contribuent à générer des recherches transdisciplinaires, à la croisée des « humanités » et des sciences sociales. Ensuite, historiquement, la postcolonial theory est aussi l'héritière de champs d'études qui se sont développés à partir des années 1960 en rapport avec les mouvements de décolonisation et d'émancipation. C'est ainsi que sont nées ce que l'on a appelé les Africana studies. Elles étaient l'expression (ou l'instrument) d'une double stratégie d'affirmation et de résistance institutionnelle de la part de l'élite minoritaire noire américaine qui avait accédé aux études supérieures. Avec les Africana studies et en leur sein, il s'agissait pour les «Afro-Américains » qui en étaient à la fois les sujets et les objets, de poursuivre un travail d'émancipation, de " décolonisation » intérieure. Leur démarche était donc symétriquement inverse de celle des area studies, que Said a pu qualifier de néo-colonialistes, avec quelque raison. Là encore, il faut souligner la dimension « performative » et non pas seulement « constative » de ce genre de programme de recherches. Il ne s'agit pas seulement d'explorer un continent resté «noir» parce que ignoré et méprisé, ni de donner ses lettres de noblesse épistémologique à un domaine d'investigations tout neuf. Ces programmes contribuent aussi à transformer les pratiques sociales et culturelles à l'échelle du continent américain et à l'échelle mondiale, puisque le « savoir» dispensé dans et par l'Université ne connaît pas de frontières (du moins en théorie).

À partir des années 1970, les women's studies répondront elles aussi à une double impulsion, « analytique » et « politique ». Cette deuxième génération de studies a pu trouver dans les travaux de Michel Foucault une justification théorique supplémentaire. Foucault a montré le rôle des «formations discursives» et des institutions qui les relaient dans la « production » de « pouvoirs ». Or, les Africana studies et les women's studies sont des « formations discursives » nouvelles qui cherchent non seulement à modifier l'équilibre des pouvoirs en place, mais à «empower » 
ses participants et ses objets, à les doter de nouveaux «pouvoirs »- au triple sens de capacité, de puissance et d'instances d'action ou de décision. La pensée de Judith Butler, par exemple, montre bien l'importance de ces notions de «power» et d'«empowerment».

LABYRINTHE - Cette évolution interne aux universités est donc en relation directe avec celle de l'ensemble de la société américaine vers le «multiculturalisme».

ANNE BERGER - Le mouvement de révolte des Noirs américains contre la ségrégation s'est divisé en trois tendances dès les années 1960 : une tendance « intégrationniste » et proto-libérale, incarnée par Martin Luther King, une tendance « révolutionnaire », d'inspiration marxiste, incarnée par les Black Panthers et une tendance « séparatiste » et « nationaliste », illustrée en partie par la conversion d'un certain nombre de Noirs américains à l'islam (à la suite de Malcolm X). Être Noir américain musulman, c'était récuser l'Amérique des WASPS (White AngloSaxon Protestants). C'était aussi, pensait-on, effectuer un retour symbolique à l'Afrique de l'origine, en rompant avec la culture dominante, en changeant de culture. Les Africana studies sont les héritières de cette histoire. Il est intéressant de noter qu'à Cornell, par exemple, mais il en va sans doute ainsi de la plupart des grands centres de recherches dévolus à ce domaine d'études, la composante africaine et africaniste est très importante. Beaucoup de chercheurs africains travaillent dorénavant au sein de ces formations. Cela témoigne peut-être en effet d'un tournant « culturaliste » dans le traitement de ces questions. Les problèmes de définition identitaire semblent l'avoir emporté sur les luttes contre la domination économique et sociale des Blancs. Mais c'est aussi, en même temps, une manière de traverser les frontières, de faire collaborer à une cause de décolonisation commune des mondes différents.

LABYRINTHE - N'est-ce pas surtout une façon de se laisser enfermer dans une catégorie?

ANNE BERGER - Peut-être, mais c'est alors une catégorie qu'on a soimême en partie créée. Le nom même d'Africana studies est tout à fait «parlant» à cet égard. Comme le fait remarquer James Siegel, anthropologue de Cornell, «Africana studies », ce n'est pas «Africa studies », même si le nom choisi renvoie évidemment au continent noir ; ce n'est pas non plus «Afro-American studies ». Si ce n'est un territoire, un pays, 
ou une culture, qu'est-ce qu' « Africana »? Ce n'est pas une aire culturelle, au sens géopolitique du terme ; ce n'est pas non plus le nom d'une communauté existante; c'est une utopie théorico-politique, qui indique un ailleurs de l'Amérique, qui programme un franchissement (et un affranchissement) symbolique de ses clôtures. Notez que le travail mené au sein des Africana studies a d'ailleurs contribué à engendrer la nouvelle catégorie culturelle, seule habilitée aujourd'hui à figurer dans le discours social, de l'African-American. L'African-American remplace désormais le « negro » des années d'esclavage et d'apartheid, et le «black» des mouvements radicaux des années 1960.

Le multiculturalisme, en ce sens, n'est pas forcément la somme de fondamentalismes communautaires réifiés, qui menaceraient la société d'éclatement. Et même s'il est vrai que les Africana studies ont tendance, par vocation et par histoire, à occuper une position assez excentrée au sein de l'institution universitaire, donc à se réfugier dans la marge dans laquelle on les relègue, on assiste au contraire depuis une quinzaine d'années à une poussée vers le « centre » des humanités d'un ensemble de nouveaux programmes ou domaines de recherches à caractère « ethnicisant»: les Asian-American studies, les Latin-American, Latino ou Hispanic studies, ou encore, dans une moindre mesure, les Native-American studies (études concernant les Indiens d'Amérique). Ces studies, qui s'inspirent des Africana studies aspirent aussi à rendre visibles et lisibles des «identités minoritaires ». C'est la force et la limite de ce genre de programme : ce n'est pas l'autre qu'on étudie et qu'on affirme, mais soi. Notez cependant que ces identités sont composites, et qu'elles se représentent et s'analysent comme telles. L'important, dans les Asian-American studies, c'est le trait d'union qui relie Asian à American tout en signalant une différence qui travaille l'Amérique de l'intérieur. Certaines de ces minorités (les Asian-Americans par exemple) jouissent d'un statut globalement privilégié dans la société américaine, contrairement aux African-Americans et aux NativeAmericans. L'affirmation de soi, de la priorité du «soi» sur l'autre, est par ailleurs un trait fondamental de l'ethos américain. En ce sens, l'inscription et la légitimation institutionnelles de ces divers champs et l'ouverture de l'Université à d'autres canons que ceux de la culture occidentale, loin de mettre en danger la « civilisation américaine », de la menacer de désintégration, contribuent à la renforcer. À certains égards, il n'y a pas plus « américain » que ce « et moi? » du discours « mino- 
ritaire », et pas plus assimilationniste que la « célébration », comme on dit, de la « diversité ».

LABYRINTHE - Est-ce avec cette complexification qu' apparaît la théorie postcoloniale?

ANNE BERGER - Pour bien comprendre la genèse intellectuelle et politique de la postcolonial theory, il faut revenir à Said et à son livre, Orientalism. Certes, Said est un humaniste. C'est au nom et en vue de « la communauté humaine », communauté cosmopolite imparfaitement préfigurée par l'institution universitaire américaine, qu'il prend position contre la tradition des études orientalistes. Voyez ce qu'il écrit à la page 328 : «Without "the Orient", there would be scholars, critics, intellectuals, human beings, for whom the racial, ethnic and national distinctions were less important than the common enterprise of promoting human community ${ }^{2}$.» La virulente critique de Said s'adosse donc à un idéal kantien d'universalité. Autre motif éminemment kantien, celui du rapport consubstantiel entre « savoir » et « liberté », que Said énonce à la page 327: «And what better norm for the scholar than human freedom and knowledge ${ }^{3}$ ? » Mais ce sont surtout les propositions méthodologiques de Said, figurant elles aussi à la fin du livre, qui ont ouvert la voie des postcolonial studies. Said met en garde contre les effets pervers de réification de la différence culturelle et de raidissement de la logique oppositionnelle induits par la création de la catégorie de 1 '«Orient». «What is another culture? » se demande-t-il à la page 325. Et de continuer : " Is the notion of a distinct culture (or race, or religion, or civilization) a useful one, or does it always get involved either in self-congratulation [...] or hostility and aggression [...]? Do cultural, religious and racial differences matter more than socio-economic categories, or politicohistorical ones ${ }^{4}$ ? Said ne répond pas directement à ces questions, mais il propose une nouvelle approche intellectuelle et

2. «Sans 1'“Orient", il y aurait des chercheurs, des critiques, des intellectuels, des êtres humains, pour qui les distinctions de race, d'ethnie, et de nation auraient moins d'importance que l'entreprise commune de promotion de la communauté humaine.» Cette traduction, comme les suivantes, est d'Anne Berger. 3. «Quel meilleur principe pour le chercheur que l'alliance de la liberté humaine et du savoir?»

4. «Qu'est-ce qu'une autre culture ? La notion de différence culturelle (ou religieuse, ou raciale, ou civilisationnelle) est-elle utile, ou favorise-t-elle toujours l'adoption de postures d'auto-congratulation, ou au contraire d'hostilité et d'agression? Les différences religieuses et raciales importent-elles plus que les catégories socio-économiques ou politico-historiques?» 
disciplinaire, qui évite le piège d'un essentialisme culturaliste - celui qu'il accuse les études orientalistes de représenter et de promouvoir politiquement néfaste, parce que toute distinction radicale entre « soi » et les « autres » est porteuse d'affrontements. La logique binaire est une logique de guerre: «Interesting work is most likely to be produced by scholars whose allegiance is to a discipline defined intellectually and not a "field" like Orientalism, defined either canonically, imperially or geographically ${ }^{5} »(326)$.

Contre la " géographisation » idéologique et essentialisante dont relèvent les études orientalistes et les area studies en général, Said en appelle à une « déterritorialisation » de la recherche, et à la soumission de celle-ci à des impératifs intellectuels, voire à telle ou telle logique théorique. Il donne comme exemple de ce type de travail les recherches menées par Clifford Geertz, en Indonésie mais aussi au Maroc, non en tant qu'orientaliste ou spécialiste de l'Islam, mais en tant qu'anthropologue. Un anthropologue ne s'intéresse pas à une «aire géopolitique» vaguement définie, mais à telle situation très locale, à tels contextes soigneusement différenciés. En même temps, l'horizon de sa démarche est philosophique. Chaque morceau d'ethnographie est une contribution à l'effort de penser le culturel et les rapports entre cultures. C'est d'ailleurs surtout vers les sciences sociales et l'anthropologie culturelle que Said se tourne dans sa recherche d'un nouveau modèle épistémologique, d'une nouvelle éthique de la recherche dans le traitement de l'«autre». Dans la foulée des travaux et des recommandations de Said, les postcolonial studies ont adopté un angle théorique, et non géoculturel. Et de même elles ont délaissé les anciennes préoccupations philologiques typiques de la recherche orientaliste, comme de toutes les études d'aires culturelles.

Certes, on peut reprocher à Said de tomber dans le travers qu'il décrit : à bien des égards, il fabrique lui-même un « Occident» monolithique et monomane quand il le désigne comme l'auteur coupable ou le «fauteur» d'un «Orient» fictif et uniforme. Mais son propos a indéniablement contribué à ouvrir de nouvelles perspectives.

5. «Le travail le plus intéressant a bien plus de chances d'être mené par des chercheurs qui s'inscrivent dans une discipline dont la définition est intellectuelle, et non dans un « champ » comme l’Orientalisme, défini par la tradition, les visées impériales ou la géographie.» 
LABYRINTHE - La contestation des area studies apparaît donc bien comme à l' origine de la fondation des postcolonial studies. Mais à quel niveau celles-ci se distinguent-elles des études "ethniques» évoquées précédemment?

ANNE BERGER - Par définition, la postcolonial theory n'a pas de cadre « ethnique » ou «culturel » particulier. En même temps, elle figure au menu de tous les programmes de recherches qui se sont constitués autour d'identités minoritaires, et qu'animent un désir de contester l'hégémonie culturelle occidentale, ou la fiction de son homogénéité.

Ce que j'ai appelé, en détournant un terme deleuzien, la « déterritorialisation » de la postcolonial theory, se manifeste aussi dans la distinction élaborée par les théoriciens de la postcolonialité eux-mêmes entre «decolonization discourse » (discours de décolonisation) et «postcolonial theory ». Srinivas Aravamudan, spécialiste des Lumières dont les travaux s'inscrivent dans la mouvance postcoloniale, résume très bien cette distinction dans son ouvrage, Tropicopolitans ${ }^{6}$. Le « decolonization discourse » est une réponse à la politique coloniale et à l'épistémologie colonialiste qui reste prise dans la logique binaire et territoriale, imposée par le colonialisme, de la lutte anticoloniale. Frantz Fanon en serait le représentant le plus accompli. Un tel « discours » a une légitimité et une pertinence historiques. Mais il risque toujours de reproduire ce qu'il dénonce : le « eux » contre «nous » se retourne en «nous » contre «eux», et on ne sort pas de la double spirale d'autocongratulation et d'hostilité dénoncée par Said. La théorie postcoloniale - du moins quand elle est au meilleur d'elle-même - s'élabore et s'élève contre les binarismes réifiants : c'est-à-dire à la fois contre les avatars du culturalisme, sur son versant « positif» (comme dans le fondamentalisme identitaire d'un certain « islamisme ») ou sur son versant « négatif » (comme dans la réaction phobique d'une certaine Amérique aux atteintes de l'« autre »), et contre un historicisme trop universalisant. Par sa critique théorique et politique de tout geste d' " homogénéisation » et de «totalisation » au nom du travail infini et déstabilisant des différences, la postcolonial theory s'inscrit dans la mouvance poststructuraliste. Ainsi, même si les théoriciens de la postcolonialité ont dans leur ensemble de la sympathie pour la démarche marxiste et sa traduction tiers-mondiste, un certain nombre d'entre eux mettent en question la vision trop unifiée (donc trop

6. Tropicopolitans : Colonialism and Agency, 1688-1804, Durham, Duke University Press, 1999. 
occidentale) du tiers monde, de l'histoire coloniale, de la voie et des mécanismes de l'émancipation, développée par le matérialisme historique.

Si la théorie postcoloniale se présente d'abord elle aussi comme une forme et une pensée de la résistance à l'hégémonie occidentale, elle récuse les logiques et les rhétoriques oppositionnelles, réactionnelles, séparatistes, phobiques, et s'évertue, dans ses formulations, ses analyses et ses modes de lecture, à mélanger les mondes pour déstabiliser sinon déplacer les frontières. D'où le privilège que la théorie postcoloniale accorde à des concepts et des pratiques théoriques comme la transculturation, la traduction, l'hybridité. Voyez comment Gayatri Spivak, par exemple, croise toujours un texte « français » ou « anglais » avec un texte ou un point de vue du Maghreb ou de l'Inde. On comprend aussi l'importance de la pensée derridienne dans l'espace théorique de la postcolonialité. Car même si bon nombre de théoriciens ne s'en réclament pas, ils (et elles) se livrent à une double déconstruction du discours colonial et du "discours décolonisateur », en interrogeant les frontières, la genèse et les modalités de l'opposition entre soi et l'autre, entre intérieur et extérieur.

\section{LABYRINTHE - Comment cela peut-il se traduire institutionnel- lement?}

ANNE BERGER - Institutionnellement, cela se traduit par le fait que la postcolonial theory, comme je l'ai dit, ne s'est pas constituée en champ de recherches séparé et autonome. Elle cherche à altérer, à diviser (dans le bon sens du terme) les disciplines existantes de l'intérieur. Elle est très présente dans les départements de littérature et d'études culturelles (tels que English, Romance studies, Asian studies, etc.), comme dans les programmes «ethniques » ou encore les départements d'anthropologie.

Il est vrai que la postcolonial theory, à la suite du «decolonization discourse », et comme les minority studies, crée un espace de parole pour les anciens colonisés et autres « subalternes ». Les principaux représentants de ce courant (Said, Homi Bhabba, Gayatri Spivak et bien d'autres encore) sont issus des anciens empires coloniaux. Ce n'est pas seulement à ce titre qu'ils s'expriment, mais c'est ce qui leur donne d'emblée une légitimité. La dominante « indienne », donc aussi anglophone, des études postcoloniales américaines est bien sûr un effet de l'importance de l'Inde dans le dispositif colonial britannique. En même temps, la présence, le statut privilégié et le travail de ces intellectuels au sein de l'institution 
américaine compliquent, voire modifient la donne historique et identitaire. Il y aurait beaucoup à dire sur le parcours de ces intellectuels, et sur ce qu'a produit leur sortie de l'Empire britannique et leur « conquête » de l'empire universitaire américain. En tout cas, il y a un rapport étroit entre leur pratique théorique de traversée des frontières et leur situation concrète dans l'institution américaine. S'ils « répondent » à l'Occident, c'est du sein même de l'Occident, et avec les armes intellectuelles que celui-ci leur a fournies. Cela dit, tous ceux qui se réclament de la postcolonial theory n'émanent pas, loin s'en faut, de populations anciennement « colonisées ». Sa contestation du principe d'identité en fait un champ très ouvert, aux acteurs plus divers que dans des programmes tels qu'Africana Studies.

LABYRINTHE - Le préfixe post serait en somme plus important que le terme colonial.

Anne Berger - Il est aussi important. Comme l'écrit Srinivas Aravamudan, le post de « postcolonial » correspond moins à une réalité qu'à une visée... Il vise l'ailleurs et l'avenir, ces fictions sans lesquelles nous ne saurions penser le monde ni le transformer. Reste que la réalité affrontée par les chercheurs dont nous parlons est une réalité marquée par les effets persistants du colonialisme.

LABYRINTHE - Mais une autre ambiguïté apparaît alors : post-colonial peut supposer une perspective diachronique, un simple effort de périodisation historique. N'est-ce pas pour cette raison qu'émergent aujourd' hui des labels comme trans-colonial ou, mieux, trans-national?

ANNE BERGER - Le post inscrit de l'histoire, de la temporalité. Mais les penseurs de la postcolonialité ne sont pas des idéalistes naîfs, ni des tenants de la fin de l'Histoire. Ils ne croient pas que le temps de l'aprèscolonialisme est venu, ni avec les guerres d'indépendance, ni aujourd'hui. En même temps, le terme a peut-être une valeur conjuratoire, cherchant, sinon à précipiter l'avènement, du moins à réunir les conditions théoriques de ce qu'il prétend décrire.

Dans Le Monolinguisme de l'autre, Jacques Derrida rappelle que toute culture est par définition coloniale, à la fois appropriatrice et expropriatrice («culture» et «colonie» sont d'ailleurs, en latin, un seul et même mot, du latin colo, colere, cultum). On ne sort pas du colonialisme, pas par le post en tout cas. 
On peut dire, alors, comme votre question le suggère, que la visée de la théorie postcoloniale, c'est le « trans- », sinon la transe, donc le mouvement et le moment même de la traversée, du passage. C'est en effet à la faveur du développement de la théorie postcoloniale (elle-même redevable aux poststructuralismes) qu'on s'est mis à essayer de penser les rapports entre soi et l'autre (voire, entre soi et soi), entre les mondes, entre les disciplines, non plus sur l'ancien mode humaniste de l' « inter » (solidarité, internationalisme, interdisciplinarité), mais sur celui de la trans(e) (altération ou différence interne, transnationalisme, transdisciplinarité). Le ou la trans(e) ne laisse pas intactes les identités des uns et des autres.

Le terme de « transcolonialité », qui saisit le phénomène colonial sans se limiter à tel ou tel cadre géopolitique, me paraît plus récent que l'adjectif «transnational », qui est très vite apparu dans la mouvance postcoloniale. La théorie postcoloniale a en particulier profondément affecté la théorie féministe et les programmes de recherches qui touchent à celleci. Le «mariage » de la théorie féministe et de la théorie postcoloniale a donné le jour à ce qu'on appelle le « féminisme transnational ». Selon les tenantes du féminisme transnational - et là encore, on peut noter une certaine dominante «indienne» de ses principales représentantes (je songe, parmi beaucoup d'autres, à Gayatri Spivak, Chandra Mohanti, ou encore Ranjana Khanna) -, le féminisme occidental, quand il ne les néglige pas, propose une vision trop homogène ( « orientalisante », si vous voulez) de l'oppression des femmes du tiers monde. Le «féminisme transnational » est plus un champ de questions qu'un courant de pensée. Il rassemble des chercheuses et des chercheurs de "sensibilités théoriques» très différentes. À Cornell, l'émergence de ce champ de questions a donné lieu à la création d'un programme qui dépend des anciennes Women's Studies (aujourd'hui rebaptisées Feminist, Gender and Sexualities Studies), mais qui a ses activités propres: Gender and Global Change. Prenez l'épineuse question du voile, un vrai cas d'école en matière de théorie féministe. Comment faire pour que la condamnation du voilement des femmes dans le monde islamique n'apparaisse pas comme la prolongation d'un discours colonial, quand on sait par exemple que c'était un topos du discours des colons (masculins de surcroît) en Algérie? Comment faire, inversement, pour éviter de cautionner, sous prétexte du respect dû à la différence des cultures, des formes violentes et historiquement déterminées de discrimination sexuelle? Il faut multiplier les précautions contextualisantes, résister à toutes les formes de tota- 
lisation ethnocentriques et ethnophiliques, etc. Il faut toute l'intelligence et le raffinement de la théorie postcoloniale et de son surgeon « féministe» pour parvenir à énoncer dans ce domaine une position juste, ouverte et nuancée.

LABYRINTHE - Une dernière question de terminologie: Gayatri Spivak, une des figures de proue de la postcolonial theory, se dit également très attachée aux subaltern studies ${ }^{7}$. Comment ces dernières viennent-elles s'intégrer dans le paysage intellectuel américain?

ANNE BERGER - Gayatri Spivak est une figure iconique de la théorie post-coloniale. Elle vient de l'Inde, mais elle a fait ses études en Angleterre, pour finalement occuper des postes dans les plus grandes universités américaines (elle enseigne actuellement à Columbia University). Elle a commencé sa carrière aux États-Unis en traduisant la Grammatologie de Derrida. Puis elle s'est radicalisée et « indianisée » (ou, si vous voulez, « contre-orientalisée »). Ce tournant sur elle-même, en quelque sorte, s'est traduit par sa décision de collaborer avec le groupe d'intellectuels et d'universitaires indiens qui ont fondé en Inde la revue Subaltern Studies, il y a environ vingt-cinq ans. Depuis, elle se déplace fréquemment entre l'Occident et le continent indien, même si sa base demeure l'université américaine. Spivak en est venue à incarner la postcolonial theory parce que son travail emblématise la jonction entre un discours anti-impérialiste, un féminisme « transnational » qui cherche à faire dialoguer son «Occident» et ses différents «Orients», et un ensemble de propositions théoriques de type poststructuraliste, largement inspiré de ce qu'on a appelé la pensée française aux États-Unis. « La postcolonialité, c'est moi », n'est-elle pas loin de proclamer.

Quant aux subaltern studies, elles n'existent pas comme telles aux États-Unis. Comme je vous l'ai dit, c'est le titre d'une revue fondée par des chercheurs indiens en histoire et sciences sociales. Post-marxistes (la notion de «subalterne» a été élaborée dans le milieu de la pensée marxiste), ces chercheurs se sont donné pour tâche, entre autres choses,

7. Selon François Cusset, les « subaltern studies » ont été lancées à Delhi en 1982 par « les historiens marxistes indiens Ranajit Guha et Partha Chatterjee ». Gayatri Spivak aurait progressivement rejoint le groupe, d'abord en publiant en 1983 un article promis à la célébrité « Can the Subaltern Speak ? ; puis en codirigeant avec R. Guha la publication de Selected Subaltern Studies, New York, Colombia University Press, 1988. Voir François Cusset, French Theory, La Découverte/Poche [2003], 2005, p. 157 [Ndlr]. 
de « désorientaliser » l'histoire de l'Inde coloniale et postcoloniale. Le rapport de Gayatri Spivak à ce groupe est un rapport de proximité critique. Mais elle a contribué à lancer - ou plutôt à relancer - le terme de «subalterne » et les problématiques qui s'y associent en publiant « Can the Subaltern Speak?», article immédiatement célèbre dans l'enceinte de l'université américaine et promis dès lors à une diffusion mondiale. Dans la mouvance des études indiennes et du procès de l'« orientalisme » entamé par Said, il s'agissait de contester l'occupation par l'Occident de la position de sujet et maître du discours. Spivak demande que l'on donne la parole aux « subalternes » et qu'on les écoute, afin que ceux et celles qui occupent historiquement la place de l'autre et de l'objet accèdent aussi au statut de sujet du discours, donc de l'Histoire. À un modèle historiographique monologique succèderaient alors des formes dialogiques et polylogiques. Il est difficile de ne pas être d'accord avec un tel projet. Mais la thèse est au fond assez simple, même si le langage de Spivak ne l'est pas.

LABYRINTHE - Son succès sur les campus est considérable...

ANNE BERGER - Gayatri Spivak tient à son statut d'icône. Pour bien comprendre ce que vous appelez son « succès », il faudrait justement faire une analyse culturelle du succès de la notion de «succès » aux ÉtatsUnis, et des mécanismes de production de la célébrité. De ce point de vue, tous ceux qui connaissent les États-Unis l'ont remarqué, l'université américaine ne diffère pas fondamentalement d'Hollywood. C'est dire aussi qu'on n'a pas besoin d'être américain(e) de souche pour le devenir. On ne naît pas américaine (donc virtuellement, «star»), on le devient.

«Américaine », Spivak l'est aussi d'une autre manière qui contribue à expliquer son succès. La puissance de l'institution universitaire américaine, sa richesse matérielle et sa capacité logistique de diffusion des idées en font un formidable lieu de répercussion de toutes les recherches menées dans le monde. Surtout, on le sait, l'Amérique est, contrairement à la France qui prétend exporter son savoir et ses «idées » et ne recevoir de leçons de personne, une importatrice avide, un lieu d'immigration et de rencontre des personnes et des discours. Les intellectuel(le)s les plus connu(e)s, au sein de l'université américaine comme dans le monde et les médias, ne sont pas les produits originaux d'une Amérique autochtone, pur cru (il y en a pourtant un certain nombre); ce sont ceux et celles qui pratiquent à une grande échelle l'importation et le mélange, le 
« digest », des idées et des œuvres qui circulent dans le monde à un moment donné. Prenez Gayatri Spivak et Judith Butler. Elles ont à cet égard quelque chose de commun. On trouve tout et tout le monde chez Spivak, à la même enseigne critique: de Kant à Heidegger et Derrida, de Freud à Mélanie Klein, de Marx à Gramsci, en passant par De Man, Agamben, Luce Irigaray, Ernesto Laclau, etc. (C'est d'ailleurs le paradoxe constitutif de la postcolonial theory, que de tirer la substance théorique de sa critique de l'hégémonie occidentale de cet Occident même, preuve que la pensée n'a décidément pas de frontières, et que l'Occident est lui-même divisé, "étrangé » de l'intérieur). De même chez Judith Butler. Si Gender Trouble, publié en 1990, a eu un tel succès, c'est aussi parce que Butler y réussit le tour de force de mettre les principales pensées et les principaux noms du moment à contribution, soit qu'elle les utilise, soit qu'elle les soumette à un traitement critique : Freud, LéviStrauss, Lacan, Derrida, Foucault, Levinas, mais encore Irigaray et Julia Kristeva, mais encore Saussure, Austin et la speech act theory, mais encore Monique Wittig et Gayle Rubin, théoriciennes d'un certain lesbianisme politique, etc. Curieusement, Hélène Cixous n'y figure pas, mais j'imagine que c'est parce qu'elle est trop « littéraire » au goût très théoricien de Butler. Une telle manière de procéder, de faire feu et forum de tout bois, sans distinction de valeur, sans souci d'incompatibilité philosophique, n'est pas concevable en France, où les intellectuels se lisent peu, se rencontrent peu les uns les autres et cherchent à maintenir une ligne ou un chemin « originaux».

Judith Butler est entrée sur la scène intellectuelle environ vingt ans après la naissance des women's studies, à un moment où la feminist theory était déjà considérée comme constituée, avec ses textes et ses débats « canoniques ». Son intervention dans le champ de la feminist theory a eu d'emblée une visée critique. En radicalisant les questions touchant aux usages, aux déterminations, à l'identité et à la normativité de la notion de « femme », non seulement dans l'espace social, mais au sein de la théorie féministe, elle a contribué à ouvrir le champ de questions rassemblées sous le nom de «queer theory». Depuis quelque temps, il me semble que c'est plutôt à une nouvelle articulation des gender studies et des postcolonial studies qu'elle travaille, en cherchant, dans le double parage de la pensée levinasienne et de la réflexion éthico-politique qu'elle mène autour du conflit israelo-palestinien, à formuler ce que j'appellerais un «féminisme de l'autre », en référence au livre de Levinas 
(Humanisme de l'autre homme): un féminisme non identitaire, qui ne réclame pas les droits ou la reconnaissance «pour soi», mais qui se préoccupe de l'autre, de l'étranger(e), qui pense et agit en vue de l'autre, sans préjuger de la nature de cet «autre» («homme», «femme» ou «autre»). Dans le contexte politique actuel, cette démarche a sa nécessité.

LABYRINTHE - Ce parcours suppose donc un rapport étroit entre ce qui s'est passé du côté des gender studies et l'évolution du paysage intellectuel américain sur la question coloniale (le decolonization discourse à la Frantz Fanon, puis son déplacement par la postcolonial theory).

ANNE BERGER - Oui, même si les sujets de ces champs d'étude sont différents, leurs modes de pensée se touchent et se comprennent. Dans l'évolution des gender studies vers la queer theory, il s'est produit quelque chose de similaire à la transformation du decolonization discourse en postcolonial theory. On a créé des women's studies au début des années 1970 dans la foulée des mouvements d'émancipation féministes - et notamment à Cornell, l'une des universités pionnières dans ce domaine. C'est l'époque où les femmes commençaient à accéder en nombre aux postes d'enseignement du supérieur, tout en rencontrant des difficultés de titularisation. Les mouvements de réflexion sur la politique et la place sociale de l'homosexualité, qui se sont développés à la faveur de la « révolution sexuelle » qui secouait alors une partie de l'Occident ont commencé à trouver une traduction universitaire au début des années 1980, avec la création des gay and lesbian studies. Situés au départ dans la mouvance et le giron des women's studies, ces programmes se sont peu à peu autonomisés, même si beaucoup de chercheuses continuent de circuler de l'un à l'autre. Si la queer theory, comme la postcolonial theory, ne s'est pas traduite institutionnellement par la création de programmes de recherches à elle exclusivement consacrés, elle a contribué à infléchir la direction des women's studies et des gay and lesbian studies. En contestant la logique binaire du discours normatif sur la sexualité, qui divise les êtres humains entre « hommes » et « femmes » et les pratiques sexuelles entre homosexualité et hétérosexualité, en privilégiant philosophiquement et politiquement toutes les formes de crossing, autrement dit, de traversée des frontières sexuelles, la queer theory a introduit le «trans- » et la transe dans les gender studies. Elle a aussi, du même coup, contribué à resexualiser (mais autrement) un discours sur les « genres » dont l'accent sociologique faisait oublier son articulation 
à la question du " désir », des politiques et des fantasmatiques de ce dernier. Sous l'impulsion de la queer theory, les gay and lesbian studies sont donc devenues des lesbian, bisexual, gay and transgender studies, dans un geste de démultiplication définitionnelle et de brouillage des orientations qui met en question la stabilité des catégories ainsi répertoriées. Pour faire partie de ce programme d'études, un individu n'a pas besoin de souscrire à l'une ou l'autre de ces catégories. On peut parfaitement être « hétérosexuel » (mais qu'est-ce que cela veut dire ?) et participer activement, voire très officiellement, à ce domaine de recherches. De même, à Cornell, les Women's Studies sont devenues il y a six ou sept ans les Feminist, Gender and Sexualities Studies. On constate dans cette nouvelle désignation une semblable volonté de déplier et de démultiplier les questions et les objets à l'intérieur de ce champ d'études (notez le pluriel de " sexualités », qui renvoie à la division, ou la multiplication, des « orientations » et des « identités » sexuelles). En ce sens aussi, on peut dire que la queer theory ne se veut pas seulement une nouvelle herméneutique de la sexualité et de la politique des sexes, mais une opératrice de trans/formations. D'adjectif (le mot veut dire « bizarre »), l'épithète «queer » est d'ailleurs devenu un verbe d'action. Queer theory keeps queering its objects and queering itself at the same time. Laissons cet énoncé en anglais.

LABYRINTHE - Que sont exactement ces "programmes » d'un point de vue institutionnel ? Et dans quelle mesure jouent-ils un rôle structurel, inhérent au fonctionnement même des universités américaines, dans cette production effrénée de nouveaux labels?

ANNE BERGER - L'institution universitaire américaine a une grande souplesse de fonctionnement. Elle invente plus facilement des disciplines, elle accueille et légitime des champs de recherches plus rapidement que sa collègue européenne. Les « programmes » n'ont pas véritablement leur équivalent en France: ce sont des centres de recherches pluridisciplinaires, voire transdisciplinaires, auxquels participent des enseignants issus de différents départements. Mais ces centres ont euxmêmes une véritable autonomie institutionnelle puisqu'ils délivrent des diplômes (maîtrise, doctorat, moins souvent licence, mais c'est possible...). Tous délivrent aussi des certificats de «mineure » (minor), ou de « concentration », attestant d'un parcours suffisant de l'étudiant(e) dans ce domaine pour qu'il soit reconnu officiellement. Tous les programmes 
que j'ai mentionnés, à commencer par les Women's Studies et leurs avatars contemporains, fonctionnent d'ailleurs de manière de plus en plus indépendante: ils recrutent désormais leurs propres professeurs, qui constituent aujourd'hui près de la moitié de leur personnel enseignant et chercheur - l'autre moitié émanant toujours de départements annexes. Bon nombre d'enseignants-chercheurs de l'Université appartiennent à plusieurs formations doctorales à la fois, sans hiérarchie d'importance ou de participation.

Moi-même, à Cornell, j'appartiens à la formation doctorale d'Études romanes, mais aussi à celle de Littérature comparée, au programme de Feminist, Gender, and Sexualities Studies, et aux Lesbian, Gay, Bisexual and Transgender Studies. Et ce n'est pas une question d'orientation sexuelle. De même, un certain nombre d'hommes travaillent au sein des FGSS (anciennes Women's Studies). Cela signifie qu'on peut diriger des thèses ou conseiller les étudiants dans ces domaines, et qu'on reconnaît la compétence des chercheurs impliqués en la matière. La souplesse de ce fonctionnement permet des rencontres fertiles, et favorise le renouvellement des perspectives.

LABYRINTHE - Certes, mais ces programmes, même dotés de nouveaux noms, supposent quand même, toujours, un fractionnement entre différentes composantes identitaires.

ANNE BERGER - Bien sûr, cette démultiplication des identités ou des topiques touchant au sexe est ambiguë. C'est un équivalent de ce qu'on appelle le multiculturalisme. On rejoindrait là une tendance fondamentale de la culture américaine: le libéralisme, au sens idéologique du terme. Ce serait une façon de mettre en pratique le principe selon lequel « chacun est le bienvenu », « il y a de la place pour tout le monde », et de réconcilier la mise en question poststructuraliste des identités et du principe d'identité avec le «principe » américain de l'affirmation individuelle. Au sein de ces formations, et précisément à cause de leur ouverture et de leur souplesse, il y a souvent des individus de sensibilités théoriques et politiques, voire culturelles et religieuses très différentes. Ces gens-là ne s'aiment pas forcément, mais ils se parlent, ils travaillent ensemble; ils s'accordent sur un devoir de solidarité institutionnelle, et ils apprennent les uns des autres, bon gré mal gré. Ou au moins ils se tiennent au courant de leurs recherches respectives. Il me semble que cela a du bon. 
LABYRINTHE - Ce très beau dépassement vers le libéralisme ne restet-il pas une sorte d' utopie strictement interne aux universités?

ANNE BERGER - C'est soulever la question, fondamentale, du rapport de l'institution universitaire avec la société. Or, elle se pose de façon très spécifique aux États-Unis. Les campus sont isolés: Cornell se situe par exemple en surplomb d'une petite ville (Ithaca), qui se trouve elle-même au milieu de rien. Et cet isolement n'est pas seulement géographique. Même les rares universités qui sont au centre des villes, comme UCLA, Columbia et New York University, sont des microcosmes largement indépendants du milieu urbain. On pourrait y voir l'expression d'une forme de solipsisme institutionnel. Et il est vrai que l'architecture des campus les plus anciens évoque sinon la «tour d'ivoire », du moins le modèle combiné du château et de l'abbaye. J'y vois surtout la mise en œuvre d'un projet kantien. Dans son essai, «Qu'est-ce que les Lumières? », Kant associe la diffusion des Lumières, autrement dit d'un savoir librement acquis, loin des vérités révélées et reçues, à ce qu'il appelle « l'usage public de la raison ». Le modèle idéal de cet « usage public de la raison » selon lui, c'est celui de la communauté sans liens et quasiment sans lieu, formée par le savant ou le philosophe avec ses lecteurs. Il distingue l'usage public de la raison de son usage « privé ». Est « privé » selon Kant, donc limité et limitatif, tout usage de la raison qui s'inscrit dans le cadre de l'exercice de fonctions professionnelles qui obligent l'individu à un devoir de réserve et de soumission à l'institution sociale dont il relève (État ou Église par exemple). Ainsi, un fonctionnaire qui s'exprime en tant que tel ne peut et ne doit faire qu'un usage « privé » de sa raison; c'est seulement s'il en fait et là où il en fait un usage libre de toute contrainte institutionnelle, dans le cadre d'un forum d'échanges informel, que cet usage autonome et potentiellement critique peut être dit « public ». En s'isolant de la sphère des relations sociales et politiques ordinaires, en proclamant son autonomie à leur égard, l'institution universitaire américaine a cherché à créer artificiellement les conditions de « l'usage public de la raison ». Bien sûr, l'Université est elle-même une institution, avec ses règles et ses contraintes. Mais c'est une institution paradoxale. La notion d'« academic freedom » (« liberté universitaire»), véritable principe du droit universitaire américain qui garantit la liberté de pensée et d'expression au sein de l'Université, signifie à la fois que celle-ci est un lieu de liberté et que la liberté est « académique », autrement dit que ce lieu seul est son lieu, la forme et l'espace 
circonscrit de son déploiement sans limite. L'Université est en ce sens un lieu d'exception (donc un lieu sans lieu), une zone franche, le sanctuaire de la « liberté ».

Certes, il arrive périodiquement que ce sanctuaire soit violé, que tel ou tel pouvoir politique fasse valoir son droit de force. C'est le sort de tous les sanctuaires. À l'époque du maccarthysme, sous la pression conjuguée du gouvernement et de l'«opinion », on a congédié certains professeurs, accusés de se livrer à des activités subversives parce qu'ils tenaient des propos taxés de « communistes ». Depuis le 11 Septembre et surtout le déclenchement de la guerre en Irak, on assiste à un retour des pratiques maccarthystes: fichage des enseignants par le FBI, menaces, procès public intenté aux individus qui s'élèvent contre la politique menée par l'administration Bush, pressions diverses exercées non seulement sur les individus, mais sur les programmes et les administrations universitaires, etc. Mais c'est bien parce que, historiquement, structurellement, et philosophiquement, l'université américaine répond à cet impératif kantien de liberté critique, parce qu'elle est, de ce fait, aux États-Unis le lieu privilégié, autoprotecteur sinon protégé, de la formation et de la diffusion de « contre-discours », que cela se passe ainsi. La prolifération de ce que vous appelez les studies, dans leur diversité, est aussi une manifestation et une conséquence de cette tradition. Enfin, le caractère foncièrement anti-intellectuel et même à bien des égards anti«scientifique» de la « culture » américaine en général - je distingue ici le respect de la «science » et l'engouement pour la technologie -, contribue à accentuer la séparation de l'Université et de la société.

LABYRINTHE - Cette indépendance kantienne a tout de même de sérieuses limites. La soumission des universités à des pressions d'ordre économique suffit à relativiser leur autonomie. Leurs innovations sont aussi orientées par la nécessité d'attirer de nouveaux étudiants, éventuellement en s'adaptant à leurs "profils identitaires ». Mais qu' en estil en amont? Les universités ne sont-elles pas soumises à des subventions privées qui peuvent pousser à la création de telle ou telle study? Le lien avec la société ne fonctionne-t-il pas surtout dans ce sens-là?

ANNE BERGER - Oui, c'est vrai. Bon nombre de domaines de recherches sont tributaires de l'intérêt politico-économique que les acteurs principaux de la société américaine leur portent, même si le soutien financier des « alumni » (les anciens étudiants de l'Université) joue aussi un 
rôle capital dans la répartition des ressources. Et les universités ne sont pas dirigées par des présidents élus par le corps enseignant. Le président d'une université américaine est nommé par un board of trustees (après « consultation » des enseignants) qui tient les cordons de sa bourse et surveille sa santé économique. Ce bureau directeur fonctionne comme les conseils d'administration des entreprises. Jusqu'à présent, l'administration interne de l'Université avait une autonomie assez grande visà-vis de ses bailleurs de fonds, qui n'intervenaient guère dans les affaires «académiques». Mais c'est en train de changer. La contamination évidente et grandissante de l'Université par la « loi du marché », aura peut-être rapidement raison de sa raison kantienne. Le véritable danger vient de cette « corporatisation » croissante des universités, qui entraîne une progressive mise au pas de leurs membres, d'autant plus subtile qu'elle ne prend pas la forme d'une pression idéologique: la compétition marchande entre les universités produit plus sûrement des effets d'uniformisation que telle censure exercée au nom d'une idéologie.

Néanmoins, et sans qu'il s'agisse pour autant de faire l'apologie du système américain, il faut bien reconnaître que, pour l'instant du moins, aucun autre système universitaire au monde n'est aussi accueillant. Non seulement le développement et la coexistence de toutes les studies dont nous avons parlé en sont une preuve, mais tout ce qui se fait, tout ce qui trouve place dans l'enceinte de l'université américaine a, comme vous le savez, des répercussions mondiales. C'est sans doute un effet de la «puissance de frappe » américaine; mais c'est peut-être aussi un signe que le dispositif kantien de «publicité » de la raison critique a fonctionné.

Certes, il existe peu d'intellectuels « publics » aux États-Unis, autrement dit peu de voix qui se font entendre au-delà du cercle universitaire: elles sont bien répercutées par une certaine presse écrite comme le New York Times, le New Yorker, Harper's, et par des hebdomadaires de gauche comme The Nation, mais leur influence pénètre difficilement le tissu de la société américaine. Et même les figures les plus célèbres, comme celles d'Edward Said ou de Judith Butler, qui tend à occuper aujourd'hui la place laissée vide par Said, sont peu connues hors des campus. Quelqu'un comme Noam Chomsky est un peu comme Woody Allen : plus célèbre en Europe qu'aux États-Unis. Mais précisément, si l'audience de ces intellectuels demeure très limitée sur le continent américain, ce qu'ils disent et font a des répercussions dans des lieux qui, en Asie ou en Europe, sont plus réceptifs à la volonté de penser le monde pour le transformer. 
LABYRINTHE - Peut-être convient-il enfin, dans cette présentation des studies, de faire la part des effets de mode et de leurs éventuelles limites: assiste-t-on à une véritable restructuration du champ universitaire? Ou les nouveaux axes d'études dégagés vont-ils, à terme, se trouver réassimilés par les disciplines traditionnelles, comme la philosophie ou la littérature comparée?

ANNE BERGER - La philosophie a une autre histoire, une autre définition aux États-Unis que son homonyme européen : son enseignement et son étude se divisent entre la philosophie analytique et la philosophie dite continentale (qui va en gros de Platon à Jacques Derrida). Dans la majorité des départements de philosophie, on enseigne surtout la philosophie analytique, et aussi ce qu'on appelle la philosophie morale, qui touche à la théologie : le primat de la philosophie analytique conduit les philosophes de profession à dialoguer plutôt avec les «scientifiques»: mathématiciens, logiciens, chercheurs en sciences cognitives, ou encore linguistes, même si la linguistique en question n'est bien sûr pas une linguistique du texte ou de l'énonciation, susceptible d'applications à des œuvres littéraires. C'est par les départements de littérature que passe l'enseignement de la philosophie « continentale », de Kant à Heidegger, de Rousseau à Derrida. Les départements de philosophie ne sont donc guère touchés ni concernés par les développements intellectuels dont nous avons parlé.

Inversement, et puisque vous évoquez le cas de la littérature comparée, je dirai que les disciplines littéraires et la littérature comparée en particulier, ont été profondément affectées par l'ouverture de ces nouveaux chantiers intellectuels. Est-ce parce que les littéraires, sont, comme la littérature qu'ils étudient, plus ouverts à l'invention, plus libres d'esprit? Est-ce parce que l'époque de la littérature comme institution occidentale du livre touche à son terme? En tout cas, la réorientation de ces disciplines se traduit de plusieurs manières, qui mériteraient chacune un long commentaire; je me contenterai de les énumérer: on assiste à une secondarisation, voire à un abandon, des questions philologiques, traditionnelles ou modernes. Le travail qu'on y fait ne relève plus, ou plus seulement, de ce qu'on a appelé la critique littéraire, ancienne ou nouvelle manière (d'où le privilège accordé au terme de "theory »). Enfin, et pour parler de la littérature comparée, la constitution de nouveaux foyers de recherche et d'intérêt est en train de renouveler cette discipline. La littérature comparée est, par origine et tradition, une disci- 
pline européenne et européaniste. Les enseignants et étudiants chercheurs dans cette discipline aux États-Unis considèrent aujourd'hui qu'elle ne peut plus et ne doit plus demeurer « occidentalocentrée ». Aussi les principaux départements de littérature comparée se sont-ils lancés dans des campagnes de recrutement qui visent à élargir le champ des littératures et cultures étudiées. Dans les décades à venir, on va surtout chercher à recruter des non-européanistes : des spécialistes de littérature(s) indienne(s), par exemple, ou chinoise, ou japonaise, ou encore « asio-américaine», etc. En ce sens, l'influence des courants qui aboutissent à la constitution des programmes de recherches dont nous avons parlé, et celle de la postcolonial theory en particulier, me parait durable.

LABYRINTHE - Des champs d'études comme les gender studies ou la postcolonial theory sont-il alors appelés à constituer des objets et des modes de savoir établis?

ANNE BERGER - L'histoire des disciplines universitaires en Occident montre que les remaniements de frontières disciplinaires sont constants. Tout cela va donc changer de forme à un moment ou à un autre, mais les disciplines des humanités et un certain nombre de disciplines relevant des sciences sociales (à l'exception de la sociologie et de la psychologie qui demeurent a-théoriques, empiriques et statisticiennes) en ont été assez profondément affectées. Je viens de vous en donner un exemple. Je crois qu'une certaine mise en question du privilège de l'Occident (j'ai évoqué Said, mais il faudrait aussi mentionner le travail de Derrida sur la « clôture métaphysique occidentale ») depuis l'Occident cosmopolite de l'université américaine porte durablement ses fruits. Il faut dire aussi - et ce n'est bien sûr pas un hasard - que cette contestation de l'hégémonie occidentale du sein même d'une institution d'origine européenne, située en extrême-Occident et ouverte par vocation à tous les mondes et tous les vents (je parle de l'université américaine), se produit à une époque que caractérise le déclin politique et économique de l'Occident. On peut donc y voir, sans le regretter nécessairement, l'un des signes, l'une des manifestations de ce déclin. Cela dit, on constate aujourd'hui un retour à la grande tradition philosophique européenne dans certains segments des départements de littérature. Le theory group animé depuis quelques années par les étudiants du département d'anglais de Cornell, par exemple, s'intéresse plus à Kant, Spinoza, Deleuze et Badiou, qu'au «canon » féministe ou postcolonial (ou aux lectures «féministes » qu'on 
peut faire de ces textes). Comme si la pure «théorie » tendait naturellement vers ce que Derrida a nommé le «phallogocentrisme » de la métaphysique occidentale. Tout est donc toujours à recommencer.

J'ai insisté sur le fait que postcolonial theory et queer theory infléchissent le discours et renouvellent les objets des humanités et des sciences sociales sans que cela se traduise par la création de programmes ou de centres de recherches autonomes. De même, on n'est pas obligé d'être affilié institutionnellement aux gender studies pour aborder dans son enseignement ou ses recherches des questions et des problématiques qui en relèvent. La plupart des départements d'études littéraires et culturelles des grandes universités américaines ont leur composante « postcoloniale », féministe et queer. Néanmoins, ces domaines d'études n'auraient pas le même impact s'ils ne s'appuyaient pas sur des relais institutionnels forts. Outre que la création des women's/gender studies a été suivie de la mise en place d'un certain nombre de dispositifs institutionnels pour assurer ou promouvoir l'égalité et la diversité des « genres » à tous les niveaux de la vie universitaire, la plupart des chercheuses et chercheurs qui s'intéressent à ces domaines fonctionnent sur le modèle de la double, voire de la multi-affiliation. Lorsque mon enseignement de littérature porte sur des questions qui touchent à la feminist theory ou à la queer theory, mes cours sont comptabilisés en Études romanes et en Feminist, Gender and Sexualities Studies. Lorsque mes cours de feminist theory ont une composante « française » importante, c'est l'inverse qui se produit. De même, si j'enseigne un cours à la croisée de la littérature et de l'anthropologie, celui-ci sera reconnu dans les deux disciplines. Une telle pratique permet le dialogue et le désenclavement des disciplines. Elle favorise le mélange des publics. Je crois beaucoup à ce modèle pédagogique et institutionnel. Le va-et-vient entre les structures et les champs de recherche me paraît très important. Il faut qu'on puisse à la fois s'inscrire dans un champ reconnu institutionnellement, s'en réclamer, le faire reconnaître par des diplômes, pousser la recherche dans ces domaines en s'appuyant sur cette reconnaissance, et qu'en même temps, on ne se laisse pas enfermer ni réduire à une approche ou une discipline. La banalisation des études féministes ou féminines dans l'université américaine, la reconnaissance de leur validité et de leur nécessité ont eu un effet tout à fait positif sur les relations collégiales entre hommes et femmes, mais aussi entre femmes et femmes. Même s'il y a toujours quelques réfractaires, une véritable culture «fémi- 
niste », sans contrainte ni affectation, s'est développée entre les membres de la communauté universitaire.

LABYRINTHE - Compte tenu de votre double appartenance au champ américain et au champ hexagonal, quel regard portez-vous sur l' université française? Et quel rôle votre situation intermédiaire vous permetelle de jouer dans ses évolutions?

ANNE BERGER - Je bénéficie d'une situation assez exceptionnelle puisque j'enseigne en effet régulièrement des deux côtés de l'Atlantique. J'ai de plus en plus conscience de mon rôle de «passeur» (ou de passeuse), aussi modeste soit-il. En tant que professeur associée à Paris VIII, j'essaie d'initier le public universitaire français aux questions que nous avons évoquées. La liberté de circulation qui m'est accordée par l'université américaine (et dont je lui suis infiniment redevable) a contribué à forger mon indépendance d'esprit et de vue. Je ne me sens identifiée ni au modèle français ni au modèle américain. Je ne crois donc pas qu'il faille importer le modèle institutionnel et disciplinaire américain. L'université française a sa propre histoire, dont elle doit assumer l'héritage, au demeurant très riche; les rapports entre État et institution scolaire sont très différents, et il faut en tenir compte.

Mais je déplore tout de même le provincialisme actuel de la France, sa suffisance et son manque d'ouverture au monde. Vue d'Amérique, c'est une province qui semble, hélas! de plus en plus petite. Est-ce parce que la France a toujours été une exportatrice de biens intellectuels et non une importatrice qu'elle s'intéresse peu ou mal à ce qui se fait ailleurs? Encore une fois, la grande force des États-Unis, c'est d'être un pays de migration, d'importation et de commerce d'idées, et pas seulement de marchandises.

Ce qui m'inquiète aussi, c'est le retour des vieilles pratiques et des vieilles distinctions hiérarchiques au sein de l'université française. Le mandarinat relève la tête, et avec lui toutes les formes de conservatisme intellectuel.

Prenez la question des gender studies. Je suis frappée par la méconnaissance générale et le mépris de leur visée, de leurs contenus, de leur audience et de leur réalité institutionnelle, qui règnent en France. De Badinter à Badiou, c'est le même son de cloche « chauviniste », au sens anglais comme au sens français du terme. Le Centre de recherches en études féminines de Paris VIII, auquel je suis affiliée, propose un docto- 
rat d'études féminines. Il a été fondé en 1974 par Hélène Cixous. C'était l'époque de la création des premiers programmes de ce genre aux ÉtatsUnis. La France intellectuelle et universitaire était donc en pointe. Depuis, ces programmes se sont répandus partout aux États-Unis et ailleurs dans le monde. En France, plus de trente ans après, la formation de Paris VIII demeure unique en son genre, et elle peine à survivre. Elle doit faire face à des mythes et des préjugés qui lui rendent la vie difficile. À quoi s'ajoutent une certaine mauvaise volonté des instances décisionnelles de l'institution universitaire, et des dissensions internes qui sont l'héritage de la violence encore insuffisamment pensée des querelles philosophiques et politiques qui ont divisé le mouvement des femmes en France. Et pourtant il y a une forte demande de la part des étudiant(e)s, du $1^{\text {er }}$ au $3^{\text {e }}$ cycle. Et les effets explosifs d'un certain choc « postcolonial » des « cultures » qui cohabitent dans la France contemporaine me paraissent susceptibles d'un traitement réflexif qui allierait pensée de la postcolonialité et analyse des relations entre les sexes. Ce n'est pas, comme je l'ai dit, que des différends théoriques et politiques importants ne divisent pas la communauté des chercheurs et chercheuses qui travaillent dans ces domaines aux États-Unis. Mais une solidarité institutionnelle sans faille garantit malgré tout la pérennité et la sérénité de leurs recherches et de leurs débats.

LABYRINTHE - Dans ce contexte, l'Europe offre des perspectives ... ANNE BERGER - Oui, la vraie chance de la France, si elle la saisit, passera très probablement par l'Europe..., mais les Français ont voté non à la Constitution. C'est un refus dont je peux comprendre les raisons et les principes. Cependant, vu des États-Unis et vu par nombre d'intellectuels transnationaux d'origine ou de provenance française comme moi, ce refus paraît stratégiquement et symboliquement suicidaire. Il fait le jeu de l'impérialisme américain qu'il prétend combattre, et des petits nationalismes divers. Il me semble que les Français « hexagonaux » sont trop simplement antimondialistes et pas assez altermondialistes.

LABYRINTHE - En parlant d'Europe - pour revenir à la question universitaire -, nous pensions surtout à la réforme du LMD: ne peutelle pas favoriser une certaine souplesse dans les cursus, et donc l'éclosion de programmes transversaux et interdisciplinaires - sans nécessairement les calquer sur le modèle américain? 
ANNE BERGER - Je voudrais y croire. Et c'est vrai que les échanges universitaires européens de type Erasmus sont d'excellente qualité. Les disciplines, les enseignants et les étudiants de France ont besoin de ces apports étrangers, de ces mouvements transfrontaliers, pour se renouveler, se revivifier, se remettre en quête et en question. La réforme du LMD, si elle est bien conduite, peut en effet faciliter les échanges et les passages à l'échelle européenne et mondiale. Mais certains symptômes d'étroitesse d'esprit hexagonal m'inquiètent: dans le domaine des humanités, je n'ai pas l'impression que l'université française recrute en ce moment les meilleurs éléments dont elle dispose. Je connais trop d'exemples de jeunes chercheurs et chercheuses de premier plan (il y en a encore), pleins d'allant et de curiosité, que leur « profil atypique » comme on dit ici, contraint de s'exiler... aux États-Unis, au Canada, voire en Angleterre. On n'accueille pas bien les étrangers en France, qu'ils soient étudiants ou enseignants-chercheurs, et ce n'est pas seulement faute de moyens matériels. On devrait d'ailleurs recruter des «étrangers » de tous pays dans l'enseignement supérieur, comme on le fait massivement au Royaume-Uni en ce moment, au lieu de pousser des «Français atypiques » à devenir «étrangers » par défaut. Voyez les résultats de cette entropie endogamique de l'université française: de moins en moins d'étudiants étrangers, d'Europe ou d'ailleurs, souhaitent faire des études en France, et ce n'est pas seulement à cause de la disparition progressive de la génération des grands intellectuels français des années 1960 et 1970. Je regrette de terminer sur une note aussi peu optimiste. Je compte sur vous, jeunes chercheuses et chercheurs qui m'interrogez ici, pour apporter un démenti à mes propos. 